\title{
Synthesis and Antioxidant Ability of 5-amino-1,3,4- oxadiazole Derivitives Containing 2,6-dimethoxyphenol
}

\author{
KHALID FAHAD ALI* \\ Department of Chemistry, Ibn Al-haitham, University of Baghdad, Baghdad 61023, Iraq. \\ ${ }^{*}$ Corresponding author E-mail: drkhalidfahad@yahoo.com \\ http://dx.doi.org/10.13005/ojc/310126
}

(Received: November 26, 2014; Accepted: January 07, 2015)

\begin{abstract}
4-(((4-hydroxy-3,5-dimethoxybenzyl)oxy)methyl)benzoic acid was synthesized from multisteps and converted to their corresponding hydrazide. The corresponding hydrazide was cyclized to their corresponding 5-amino-1,3,4-oxadizole. Newly Schiff bases (7a-7e) were synthesized from reaction the 5-amino-1,3,4-oxadizole with several substituted of 4hydroxybenzylaldehyde. The resulting compounds were characterized based on their IR, ${ }^{1} \mathrm{H}-$ NMR, ${ }^{13} \mathrm{C}-\mathrm{NMR}$, and HRMS data. 2,2-Diphenyl-1-picrylhydrazide (DPPH) and ferric reducing antioxidant power (FRAP) assays were used to test the antioxidant properties of the synthesized compounds. Compound $7 \mathrm{~d}$ and $7 \mathrm{e}$ exhibited significant free-radical scavenging ability in both assays.
\end{abstract}

Key words: 5-amino-1,3,4-oxadiazole, Schiff base, antioxidant, DPPH, FRAP.

\section{INTRODUCTION}

The reactive oxygen species (ROS) and other related free radicals species are capable to react either directly or indirectly, to damage all biomolecules, such as proteins, lipids, DNA and carbohydrates resulting in cell damage and subsequently this damage can cause many diseases ${ }^{1}$. Many researchers reported the implication of these free radicals will cause several diseases such as inflammatory ${ }^{2}$, cancers ${ }^{3}$, degenerative $^{4}$ and chronic diseases ${ }^{5}$. Phenolic antioxidant compounds are one of the most important antioxidant species which can inhibit the oxidative stress in biological system and prevent any damage. These compounds also show broad biological activity as anti inflammatory ${ }^{6}$ and anticancer ${ }^{7}$.

Generally, antioxidants donate protons to become stable free radicals. This stability increases with the extent of delocalization and enhances antioxidant ability ${ }^{8,9}$. As such, many synthesized compounds containing long chain resonance exhibited high antioxidant activity. Furthermore, the compounds which can be classified as a strong antioxidant usually shared common structure features. They often own multiple phenolic hydroxyl 
groups like flavonoids ${ }^{10}$ or which have full conjugation À system like carotenoids ${ }^{11}$. Moreover, exhibited substituted groups might influence on the scavenging ability. This indicates the existence of a close relationship between the chemical structure and the ability to scavenge free radicals.

Derivatives of 1,3,4-oxadiazole are shown to exhibit various types of biological activities ${ }^{12-14}$ and antioxidant ability ${ }^{9,15,16}$ and compounds of Schiff base derivatives have even broader properties and applications such as liquid crystalline ${ }^{17,18}$, nonlinear optical devices ${ }^{19}$, dyes ${ }^{20}$, biological ${ }^{21-23}$ and pharmaceutical ${ }^{24}$ applications. As for 2,6dimethoxyphenol derivatives, there has been widespread interest during the last years as antioxidant materials ${ }^{25,26} . . n$ this work we presented the synthesis of new 4-(((4-(5-((aryidene)amino)1,3,4-oxadiazol-2-yl)benzyl)oxy) methyl)-2,6dimethoxyphenol (7a-7e) as new antioxidant material.

\section{MATERIALAND METHODS}

\section{Chemistry}

The IR spectra were obtained with a Perkin Elmer 400 Fourier Transform Infrared (FTIR) Spectrometer. ${ }^{1} \mathrm{H}$ and ${ }^{13} \mathrm{C}-\mathrm{NMR}$ spectra were recorded at Joel Lambda and ECA DELTA spectrometers at $400 \mathrm{MHz}$ ) UM, Malaysia). $\mathrm{CDCl}_{3}$ and DMSO- $d_{6}$ were used as solvents with TMS as the internal standard. The mass spectra were recorded using an Agilent 5975 system for El/MS and a Finnigan TSQ7000 for HREIMs (NUS, Singapore). For UV spectroscopy, a Power Wave X340 (BIO-TEK Instruments, Inc., Winooski, VT, USA) was used to record the FRAP and DPPH. Melting points were measured on a Gallenkamp melting point apparatus in open-end capillary tubes. Flash column chromatography on silica gel 60 (230-400 mesh, E. Merck) was employed. General grade solvents and reagents were purchased from commercial suppliers and used without further purification.

\section{Synthesis of 3,5-dimethyl-4-((trimethylsilyl)} oxy)benzaldehyde (1)

4-Hydroxy-3,5-dimethylbenzaldehyde (3.64 g, $20 \mathrm{mmol}$ ) was dissolved in dry DMF (15 $\mathrm{mL}$ ) containing anhydrous potassium carbonate
( $2.76 \mathrm{~g}, 25 \mathrm{mmol})$. The mixture stirred for 30 minutes at ambient temperature, then Chlorotrimethylsilane (3.25, $30 \mathrm{mmol}$ ) was added drop wise. The stirring mixture was heated under reflux (70-80 C) for $24 \mathrm{~h}$. After cooling the resulting product was extracted from diethyl ether $(3 \times 50 \mathrm{~mL})$ and dried under calcium chloride. The crude product was purified by column chromatography using hexane-ethylacitate (8:2) as eluent to obtain colorless oil, Yield; 74\%, $\mathrm{Bp}, 288-292 \mathrm{C}$ at $760 \mathrm{mmHg}, \mathrm{d}=1.23$ at $25 \mathrm{C}$. IR (liquid film) $\mathrm{v}_{\max } 3033\left(\mathrm{CH}_{\mathrm{Ar}}\right), 2973,2958\left(\mathrm{CH}_{\text {aliphatic }}\right)$ ,1674(CO), 1595(C=C), 1190 (Ar-O-C), 842( $\left(\mathrm{Si}_{-} \mathrm{CH}_{3}\right)$ $\mathrm{cm}^{-1},{ }^{1} \mathrm{H}-\mathrm{NMR}\left(400 \mathrm{MHz}, \mathrm{CDCl}_{3}\right): \delta 0.23(\mathrm{~s}, 9 \mathrm{H}$, Si$\left.\left(\mathrm{CH}_{3}\right)_{3}\right), 3.84\left(\mathrm{~s}, 6 \mathrm{H}, 2 \times \mathrm{OCH}_{3}\right) 7.13(\mathrm{~s}, 2 \mathrm{H}, \mathrm{H}-3), 9.85(\mathrm{~s}$,

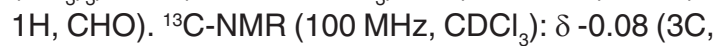
$\mathrm{Si}-\left(\mathrm{CH}_{3}\right)_{3}, 55.42\left(2 \mathrm{C}, \mathrm{OCH}_{3}\right), 108(2 \mathrm{C}, \mathrm{C}-3)$, 131.63(1C,C-4), 134.17(1C, C-3), 151.83(1C, C2), 192.07(1C, CO). HREIMs m/z = 254.0968 [MÙ+] (calc. for $\mathrm{C}_{12} \mathrm{H}_{18} \mathrm{O}_{4} \mathrm{Si}, 254.0974$ ).

\section{Synthesis of 3,5-dimethyl-4-((trimethylsilyl) oxy)benzyl alcohol (2)}

Sodium borohydride $(0.75 \mathrm{gm}, 20 \mathrm{mmol})$ was added with small portion to a stirred solution of 3,5-dimethyl-4-((trimethylsilyl)oxy)benzaldehyde (3 g, $13.3 \mathrm{mmol})$ in THF-Methanol 4:1 (25 mL) and refluxed overnight. Upon cooling distill water (50 $\mathrm{mL}$ ) was added and the mixture was stirred for further 10 minutes. The crude product was extracted from diethyl ether. The organic layer washed three times with saturated solution of Sodium hydrogen sulfate. After evaporating the solvent pale yellow oil was obtained. Yield 87\%, Bp 314-317 C at 760 $\mathrm{mmHg}, \mathrm{d}=1.12$ at $25 \mathrm{C}$. IR (liquid film) $\mathrm{v}_{\max } 3329(\mathrm{OH})$, 3067 $\left(\mathrm{CH}_{\mathrm{Ar}}\right), 2943,2877\left(\mathrm{CH}_{\text {aliphatic }}\right), 1593(\mathrm{C}=\mathrm{C})$, 1205 (Ar-O-C), 859(Si-CH $)_{3} \mathrm{~cm}^{-1},{ }^{1} \mathrm{H}-\mathrm{NMR}(400 \mathrm{MHz}$, $\left.\mathrm{CDCl}_{3}\right): \delta 0.20\left(\mathrm{~s}, 9 \mathrm{H}, \mathrm{Si}-\left(\mathrm{CH}_{3}\right)_{3}\right), 2.94(\mathrm{bs}, 1 \mathrm{H}, \mathrm{OH})$, $3.82\left(\mathrm{~s}, 6 \mathrm{H}, 2 \times \mathrm{OCH}_{3}\right), 4.51\left(\mathrm{~s}, 2 \mathrm{H}, \mathrm{OCH}_{2}\right), 6.67(\mathrm{~s}$, $2 \mathrm{H}, \mathrm{H}-3) .{ }^{13} \mathrm{C}-\mathrm{NMR}\left(100 \mathrm{MHz}, \mathrm{CDCl}_{3}\right): \delta-0.05(3 \mathrm{C}$, $\mathrm{Si}-\left(\mathrm{CH}_{3}\right)_{3}, 55.42\left(2 \mathrm{C}, \mathrm{OCH}_{3}\right), 62.34\left(1 \mathrm{C}, \mathrm{CH}_{2} \mathrm{OH}\right)$, 107.52(2C, C-3), 128.44(1C, C-1), 135.68(1C, C4), 152.08(1C, C-2), HREIMs m/z = 256.1129 [MÙ+] (calc. for $\mathrm{C}_{12} \mathrm{H}_{20} \mathrm{O}_{4} \mathrm{Si}, 256.1131$ ).

Synthesis of $4-(((3,5-d i m e t h o x y-4-$
((trimethylsilyl)oxy)benzyl)oxy) methyl)
benzonitrile (3)
Small portions $\quad$ of 4 -
(bromomethyl)benzonitrile $(2.3 \mathrm{~g}, 11.6 \mathrm{mmol})$ was (bromomethyl)benzonitrile $(2.3 \mathrm{~g}, 11.6 \mathrm{mmol})$ was
added to a stirring solution of 3,5 -dimethyl-4- 
((trimethylsilyl)oxy)benzyl alcohol (2.6 g,11.6 mmol) in pyridine $(15 \mathrm{~mL})$ within 30 minutes. After complete the addition the mixture was refluxed for $6 \mathrm{~h}$. Upon cooling the mixture poured in to crashed water (100 $\mathrm{mL}$ ) and acidified with $5 \%$ of hydrochloric acid. The product was extracted with diethyl ether $(3 \times 25 \mathrm{~mL})$ and washed with water, then dried over magnesium sulfate. After evaporating the solvent, the crude material was purified by column chromatography using hexane-ethylacetate $(9: 1)$ as eluent to obtain colorless oil which is solidify after two days to obtain white powder. Yield $61 \%, \mathrm{Mp} 36-38 \mathrm{C}$, IR (KBr) $\mathrm{v}_{\max }$ $3042\left(\mathrm{CH}_{\mathrm{Ar}}\right), 2962,2890 \quad\left(\mathrm{CH}_{\text {aliphatic }}\right)$, 2248(Ca”N),1595(C=C), 1195 (Ar-O-C), 867(Si$\left.\mathrm{CH}_{3}\right) \mathrm{cm}^{-1} .{ }^{1} \mathrm{H}-\mathrm{NMR}\left(400 \mathrm{MHz}, \mathrm{CDCl}_{3}\right): \delta 0.18(\mathrm{~s}, 9 \mathrm{H}$, $\left.\mathrm{Si}-\left(\mathrm{CH}_{3}\right)_{3}\right), 3.89\left(\mathrm{~s}, 6 \mathrm{H}, 2 \times \mathrm{OCH}_{3}\right), 4.31\left(\mathrm{~s}, 2 \mathrm{H}, \mathrm{OCH}_{2}\right)$, $4.35\left(\mathrm{~s}, 2 \mathrm{H}, \mathrm{OCH}_{2}\right), 6.62(\mathrm{~s}, 2 \mathrm{H}, \mathrm{H}-3), 7.41(\mathrm{~d}, 2 \mathrm{H}, \mathrm{J}$ 8.2, H-8), 7.49(d, 2H, J 7.9, H-9). ${ }^{13} \mathrm{C}-\mathrm{NMR}(100 \mathrm{MHz}$, $\left.\mathrm{CDCl}_{3}\right): \delta-0.046\left(3 \mathrm{C}, \mathrm{Si}-\left(\mathrm{CH}_{3}\right)_{3}, 56.02\left(2 \mathrm{C}, \mathrm{OCH}_{3}\right)\right.$, $71.14\left(1 \mathrm{C}, \mathrm{CH}_{2} \mathrm{OCH}_{2}\right), 70.67\left(1 \mathrm{C}, \mathrm{CH}_{2} \mathrm{OCH}_{2}\right)$, 101.22(1C, CN ), 107.75(2C, C-3), 127.89(1C,C1), 128.55(2C,C-8), 131.87(2C,C-9), 135.43(1C,C10), 138.44(1C-C-4), 140.2(1C-C7) 151.98(2C, C2), HREIMs $\mathrm{m} / \mathrm{z}=318.1097$ [MÙ'] (calc. for $\left.\mathrm{C}_{17} \mathrm{H}_{18} \mathrm{O}_{6}, 318.1103\right)$.

\section{Synthesis of 4-(((4-hydroxy-3,5-dimethoxybenzyl) oxy)methyl)benzoic acid (4)}

$4-(((3,5-\mathrm{d}$ i m e t h o x y - 4 ((trimethylsilyl)oxy)benzyl)oxy)methyl)benzonitrile $(2.2 \mathrm{~g}, 6 \mathrm{mmol})$ was dissolved in ethanol $(25 \mathrm{~mL})$ and $5 \mathrm{~mL}$ of $4 \mathrm{~N} \mathrm{KOH}$ solution. The mixture was refluxed for $48 \mathrm{~h}$. After evaporation the solvent the crude product extract with $20 \mathrm{~mL}$ ethyl acetate The organic layer was ignored. The aqueous layer reduced to $5 \mathrm{~mL}$ by evaporation under reduced pressure. of acetic acid-THF 1:1 (10mL) was added to the aqueous layer then refluxed overnight. The precipitated collected by filtration and washed with water. Recrystallized with ethanol to obtain white microcrystals. Yield $66 \%$, Mp 152-154 C, IR (KBr) $\mathrm{v}_{\max } 3350(\mathrm{OH}), 3042\left(\mathrm{CH}_{\mathrm{Ar}}\right), 2962,2890\left(\mathrm{CH}_{\text {aliphatic }}\right)$, 1673(C=O) 1595(C=C), 1195 (Ar-O-C), $\mathrm{cm}^{-1} \cdot{ }^{1} \mathrm{H}-$ $\operatorname{NMR}\left(400 \mathrm{MHz}, \mathrm{CDCl}_{3}\right): 3.89\left(\mathrm{~s}, 6 \mathrm{H}, 2 \times \mathrm{OCH}_{3}\right), 4.32$ $\left(\mathrm{s}, 2 \mathrm{H}, \mathrm{OCH}_{2}\right), 4.37\left(\mathrm{~s}, 2 \mathrm{H}, \mathrm{OCH}_{2}\right), 6.62(\mathrm{~s}, 2 \mathrm{H}, \mathrm{H}-$ 3), $7.62(\mathrm{~d}, 2 \mathrm{H}, \mathrm{J} 8.2, \mathrm{H}-8), 8.22(\mathrm{~d}, 2 \mathrm{H}, \mathrm{J} 8.0, \mathrm{H}-9)$. 9.12 (bs, $1 \mathrm{H}, \mathrm{OH}),{ }^{13} \mathrm{C}-\mathrm{NMR}\left(100 \mathrm{MHz}, \mathrm{CDCl}_{3}\right)$ : 56.02(2C, $\left.\mathrm{OCH}_{3}\right), 71.14\left(1 \mathrm{C}, \underline{\mathrm{CH}}_{2} \mathrm{OCH}_{2}\right), 70.67(1 \mathrm{C}$, $\left.\mathrm{CH}_{2} \mathrm{OCH}_{2}\right), 108.35(2 \mathrm{C}, \mathrm{C}-3), 128.71(2 \mathrm{C}, \mathrm{C}-8)$, 129.26(1C,C-10), 130.43(2C, C-9), 133.32(1C, C-
4), 139.44(1C, C-1), 142.08(1C, C-7) 151.98 (2C, C2), $169.6(1 \mathrm{C}, \mathrm{C}=\mathrm{O})$ HREIMs $\mathrm{m} / \mathrm{z}=318.1100$ [MÙ+] (calc. for $\mathrm{C}_{17} \mathrm{H}_{18} \mathrm{O}_{6}$ ), 318.1103).

\section{Synthesis of 4-(((4-hydroxy-3,5-dimethoxybenzyl) oxy)methyl) benzohydrazide (5)}

Thionylchloride $(4 \mathrm{~mL})$ was added in small portions of 4-(((4-hydroxy-3,5-dimethoxybenzyl) oxy)methyl)benzoic acid to $(2 \mathrm{~g}, 6.2 \mathrm{mmol})$. The mixture was refluxed for $3 \mathrm{~h}$, the excess of thionyl chloride was removed under reduced pressure. 0.1 moles of acid chloride (without further purification) were dissolved in dry benzene $(15 \mathrm{~mL})$, and it was transferred to an addition funnel hydrazine hydrate (98\%) $5 \mathrm{~mL}$ in dried benzene $(10 \mathrm{~mL})$ was added into a two neck flask that equipped with a condenser. The addition funnel was then fixed onto the flask and secured firmly. The acid chloride was added dropwise at $0 \mathrm{C}$. After that, the mixture was allowed to stand for $1 \mathrm{~h}$ at an ambient temperature. It was then stirred and refluxed for $3 \mathrm{~h}$. The excess solvent was removed under reduced pressure and the crude solid was collected, wash with water and recrystallized from ethanol afforded white solid. Yield 84\%, Mp 92-94 C, IR (KBr) $v_{\max } 3418.2(\mathrm{OH}$ phenol), 3298, $3210\left(\mathrm{NH}, \mathrm{NH}_{2}\right), 3055$ (CHAr), 2958 2880 (CHaliphatic), $1665 \quad(\mathrm{C}=\mathrm{O}), 1595(\mathrm{C}=\mathrm{C})$, 1197(Ar-O-C), cm-1. ${ }^{1}$ H-NMR (400MHz, DMSO-d $\mathrm{d}_{6}$ ): $3.68\left(\mathrm{~s}, 6 \mathrm{H}, 2 \times \mathrm{OCH}_{3}\right), 4.33\left(\mathrm{~s}, 2 \mathrm{H}, \mathrm{OCH}_{2}\right), 4.29$ (s, $\left.2 \mathrm{H}, \mathrm{OCH}_{2}\right), 4.74\left(\mathrm{bs}, 2 \mathrm{H}, \mathrm{NH}_{2}\right) 6.56(\mathrm{~s}, 2 \mathrm{H}, \mathrm{H}-3)$, $7.69(\mathrm{~d}, 2 \mathrm{H}, \mathrm{J} 8.2, \mathrm{H}-8), 8.22(\mathrm{~d}, 2 \mathrm{H}, \mathrm{J} 7.88, \mathrm{H}-$ 9), $8.83(\mathrm{bs}, 1 \mathrm{H}, \mathrm{CONH}), 9.14(\mathrm{bs}, 1 \mathrm{H}, \mathrm{OH}),{ }^{13} \mathrm{C}-\mathrm{NMR}$ $\left(100 \mathrm{MHz}, \mathrm{DMSO}-\mathrm{d}_{6}\right): 56.1\left(2 \mathrm{C}, \mathrm{OCH}_{3}\right), 71.11(1 \mathrm{C}$, $\left.\mathrm{CH}_{2} \mathrm{OCH}_{2}\right), 70.59\left(1 \mathrm{C}, \mathrm{CH}_{2} \mathrm{OCH}_{2}\right), 107.85(2 \mathrm{C}, \mathrm{C}-3)$, 129.01(2C,C-8), 128.96(1C, C-10), 130.13(2C, C9), 133.33(1C, C-4), 138.84(1C, C-1), 141.78(1C, C-7) 152.08 (2C, C-2), 166.11(C=O). HREIMs m/z $=332.1368$ [MÙ'] (calc. for $\mathrm{C}_{17} \mathrm{H}_{20} \mathrm{~N}_{2} \mathrm{O}_{5}, 332.1372$ ).

Synthesis of 4-((4-(5-amino-1,3,4-oxadiazol-2yl)benzyl)oxy)methyl)-2,6-dimeth oxyphenol (6)

A solution of hydrazide, $5(2 \mathrm{~g}, 6 \mathrm{mmol})$ in methanol $(10 \mathrm{~mL})$ and sodium bicarbonate $(0.5,6$ $\mathrm{mmol}$ ) was stirred in $50 \mathrm{~mL}$ round bottom flask, then cyanogene bromide $((0.66 \mathrm{gm}, 6.3 \mathrm{mmol})$ was added. The mixture was left stirring at ambient temperature overnight. After that, cold water $(5 \mathrm{~mL})$ was added to the mixture and the precipitate was collected and dried at $60^{\circ} \mathrm{C}$. The white solid was recrystallized from ethanol to give (62\%) yield; $\mathrm{Mp}$ 
$132-134{ }^{\circ} \mathrm{C}, \mathrm{IR}(\mathrm{KBr}) \mathrm{v}_{\max } 3468\left(\mathrm{br}, \mathrm{OH}_{\text {phenol }}\right), 3318$, $32\left(\mathrm{NH}_{2}\right), 3093\left(\mathrm{CH}_{\mathrm{Ar}}\right), 2960,2870\left(\mathrm{CH}_{\text {aliphatic }}\right)$ : 1609(C=N), 1600, 1552(C=C), 1250(C-N), $1203(\mathrm{C}-$ O), 1099 (C-O-C); cm- ${ }^{1}$; ${ }^{1} \mathrm{H}-\mathrm{NMR}$ (400MHz, DMSO$\left.\mathrm{d}_{6}\right) 3.71\left(\mathrm{~s}, 6 \mathrm{H}, 2 \times \mathrm{OCH}_{3}\right), 4.42\left(\mathrm{~s}, 2 \mathrm{H}, \mathrm{OCH}_{2}\right), 4.45$ $\left(\mathrm{s}, 2 \mathrm{H}, \mathrm{OCH}_{2}\right), 6.57(\mathrm{~s}, 2 \mathrm{H}, \mathrm{H}-3), 7.51$ (bs, $\left.2 \mathrm{H}, \mathrm{NH}_{2}\right)$ 7.72 (d, 2H, J 8.22, H-8), 8.18(d, 2H, J 8.2, H-9), 9.11(bs, $1 \mathrm{H}, \mathrm{OH}),{ }^{13} \mathrm{C}-\mathrm{NMR}\left(100 \mathrm{MHz}, \mathrm{DMSO}-\mathrm{d}_{6}\right)$ : 56.12(2C, $\left.\mathrm{OCH}_{3}\right), 71.21\left(1 \mathrm{C}, \mathrm{CH}_{2} \mathrm{OCH}_{2}\right), 69.91(1 \mathrm{C}$, $\left.\mathrm{CH}_{2} \mathrm{OCH}_{2}\right), 107.85(2 \mathrm{C}, \mathrm{C}-3), 129.06(2 \mathrm{C}, \mathrm{C}-9)$, 129.15(1C, C-10), 131.33(2C, C-8), 133.04(1C, C4), 138.75(1C, C-1), 142.07 (1C, C-7) 151.89 (2C, $\mathrm{C}-2), 158.22 \& 162.13\left(\mathrm{C}=\mathrm{N}_{\text {oxadiazole }}\right)$. HREIMs $\mathrm{m} / \mathrm{z}=$ 357.1320 [MÙ+] (calc. for $\mathrm{C}_{18} \mathrm{H}_{19} \mathrm{~N}_{3} \mathrm{O}_{5}, 357.1325$ ).

General synthesis of 4-(((4-(5-((aryidene)amino)1,3,4-oxadiazol-2-yl)benzyl)oxy) methyl)-2,6dimethoxyphenol (7a-e)

To hot solution of 4-(((4-(5-amino-1,3,4oxadiazol-2-yl)benzyl)oxy)methyl)-2,6-dimethoxy phenol $(1 \mathrm{mmol})$ in glacial acetic acid-ethanol 1:1 (20 mL), 4-hydroxy substituted benzaldehyde (1 $\mathrm{mmol})$ in ethanol $(10 \mathrm{~mL})$ were added drop wise. The mixture refluxed for $18 \mathrm{~h}$. Then reduce the solvent to half under reduced pressure. After cooling the precipitate collected, washed with cold distilled water and recrystallized from suitable solvent.

Synthesis of 4-(((4-(5-)(4-hydroxybenzylidene) amino)-1,3,4-oxadiazol-2-yl)benz yl)oxy)methyl)2,6-dimethoxyphenol (7a)

Crude product recrystallized from aqueous ethanol to produce pale yellow precipitate, Yield $72 \%, \mathrm{Mp} 179-181^{\circ} \mathrm{C}, \mathrm{IR}(\mathrm{KBr}) \mathrm{v}_{\max }, 3548\left(\mathrm{OH}_{\text {phenol }}\right)$, $3087\left(\mathrm{CH}_{\text {Aromatic }}\right), 1615(\mathrm{CH}=\mathrm{N}), 1605\left(\mathrm{C}=\mathrm{N}_{\text {Oxadiazole }}\right)$, $1600,1985(\mathrm{C}=\mathrm{C}), 1250(\mathrm{C}-\mathrm{N}), 1203(\mathrm{C}-\mathrm{O}), 1099$ (CO-C); cm- ${ }^{1},{ }^{1} \mathrm{H}-\mathrm{NMR}$ (DMSO-d $\left.{ }_{6}, \delta\right): 3.65(\mathrm{~s}, 6 \mathrm{H}, 2 \times$ $\left.\mathrm{OCH}_{3}\right), 4.40\left(\mathrm{~s}, 2 \mathrm{H}, \mathrm{OCH}_{2}\right), 4.49\left(\mathrm{~s}, 2 \mathrm{H}, \mathrm{OCH}_{2}\right), 6.62$ (s, 2H, H-3), 6.86( 2H, d, J 8.5, H-16), 7.86 (d, 4H, J 8.5,H-15), 7.91 (d, 2H, J 8.22,H-8), 8.2(d, 2H, J $8.2, \mathrm{H}-9), 8.66(\mathrm{~s}, 1 \mathrm{H}, \mathrm{CH}=\mathrm{N}), 9.19(\mathrm{bs}, 1 \mathrm{H}, \mathrm{OH}), 9.96$ (bs, $2 \mathrm{H}, \mathrm{OH}),{ }^{13} \mathrm{C}-\mathrm{NMR}\left(100 \mathrm{MHz}\right.$, DMSO-d $\mathrm{d}_{6}$ ): 56.12(2C, $\left.\mathrm{OCH}_{3}\right), 71.21\left(1 \mathrm{C}, \mathrm{CH}_{2} \mathrm{OCH}_{2}\right), 69.91(1 \mathrm{C}$, $\left.\mathrm{CH}_{2} \mathrm{OCH}_{2}\right), 107.85(2 \mathrm{C}, \mathrm{C}-3), 116.3(2 \mathrm{C}, \mathrm{C}-16, \mathrm{C}-18$ ), $125.66(1 \mathrm{C}, \mathrm{C}-10) 129.01(2 \mathrm{C}, \mathrm{C}-9), 129.15(1 \mathrm{C}, \mathrm{C}-$ 14), $130.47(2 \mathrm{C}, \mathrm{C}-15, \mathrm{C}-19), 131.33(2 \mathrm{C}, \mathrm{C}-8)$, 133.04(1C, C-4), 138.75(1C,C-1), 142.07 (1C,C-7) $151.89(2 \mathrm{C}, \mathrm{C}-2), 157(1 \mathrm{C}, \mathrm{C}-17), 160.31(\mathrm{C}=\mathrm{N})$, $160.93 \& 162.23(\mathrm{C}=\mathrm{N}$ oxadiazole $)$. HREIMs $\mathrm{m} / \mathrm{z}=$ $461.1581 \mathrm{MM}^{+}$] (calc. for $\mathrm{C}_{25} \mathrm{H}_{23} \mathrm{~N}_{3} \mathrm{O}_{6}, 461.1587$ ).
Synthesis of 4-(( 4-(5-)((4-hydroxy-3methoxybenzylidene)amino)-1,3,4-oxadiazol-2yl)benzyl)oxy)methyl)-2,6-dimethoxyphenol 7b

The crude mixture was purified by column chromatography using hexane- ethylacetate $(5: 1)$ as eluent to give a white amorphous solid. Yeild $69 \%, \mathrm{Mp} 122-124^{\circ} \mathrm{C}$. IR ( $\left.\mathrm{KBr}\right) \mathrm{v}_{\max }, 3608\left(\mathrm{OH}_{\text {phenol }}\right)$, $3083\left(\mathrm{CH}_{\mathrm{Ar}}\right), 1621(\mathrm{CH}=\mathrm{N}), 1605\left(\mathrm{C}=\mathrm{N}_{\text {oxadiazole }}\right)$, 1594, 1983(C=C), $1231(\mathrm{C}-\mathrm{N}), 1193(\mathrm{C}-\mathrm{O}), 1109$ (C$\mathrm{O}-\mathrm{C}) ; \mathrm{cm}^{-1} .{ }^{1} \mathrm{H}$ NMR (DMSO-d $\left.\mathrm{d}_{6}, \delta\right): 3.67(\mathrm{~s}, 6 \mathrm{H}, 2 \times$ $\left.\mathrm{OCH}_{3}\right), 3.88\left(\mathrm{~s}, 3 \mathrm{H}, \mathrm{OCH}_{3}\right), 4.43\left(\mathrm{~s}, 2 \mathrm{H}, \mathrm{OCH}_{2}\right), 4.55$ $\left(\mathrm{s}, 2 \mathrm{H}, \mathrm{OCH}_{2}\right), 6.62(\mathrm{~s}, 2 \mathrm{H}, \mathrm{H}-3), 6.83(\mathrm{~d}, 1 \mathrm{H}, \mathrm{J}$ $7.78, \mathrm{H}-18$ ), $6.86(2 \mathrm{H}, \mathrm{d}, \mathrm{J} 8.1, \mathrm{H}-8), 7.23$ (dd, $2 \mathrm{H}, \mathrm{J}$ $8.24 ; 1.8, \mathrm{H}-19), 7.41(\mathrm{~s}, 1 \mathrm{H}, \mathrm{H}-15)), 8.2(2 \mathrm{H}, \mathrm{d}, \mathrm{J} 8.2, \mathrm{H}-$ 9), $8.59(\mathrm{~s}, 1 \mathrm{H}, \mathrm{CH}=\mathrm{N}), 9.19(\mathrm{bs}, 1 \mathrm{H}, \mathrm{OH}), 9.96$ (bs, $2 \mathrm{H}, \mathrm{OH}) \cdot{ }^{13} \mathrm{C}-\mathrm{NMR}\left(100 \mathrm{MHz}\right.$, DMSO-d $\left.\mathrm{d}_{6}\right): 56.11(2 \mathrm{C}$, $\left.\mathrm{OCH}_{3}\right), 56.28\left(1 \mathrm{C}, \mathrm{OCH}_{3}\right) 71.21\left(1 \mathrm{C}, \mathrm{CH}_{2} \mathrm{OCH}_{2}\right)$, 69.91(1C, $\left.\quad \mathrm{CH}_{2} \mathrm{OCH}_{2}\right), 107.85(2 \mathrm{C}, \quad \mathrm{C}-3)$, 110.32(1C,C-15), 115.85 (1C,C-18), 124.22 (1C,C10), 126.0(1C, C-19), 129.01(2C, C-9), 129.15(1C, C-14), 131.33(2C, C-8), 138.75(1C, C-1), 142.07 (1C, C-4), 148.5(1C,C-7), 151.89 (2C, C-2), 153.4(1C, C-16), 157(1C, C-17), 161.30(C=N), $162.53 \& 164.22(\mathrm{C}=\mathrm{N}$ oxadiazole $)$. HREIMs $\mathrm{m} / \mathrm{z}=$ $491.1690\left[\mathrm{M}^{+}\right]$(calc. for $\mathrm{C}_{26} \mathrm{H}_{25} \mathrm{~N}_{3} \mathrm{O}_{7}, 491.1693$ ).

Synthesis of 4-(((4-)(5-)(4-hydroxy-3ethoxybenzylidene)amino)-1,3,4-oxadiazol-2yl)benzyl)oxy)methyl)-2,6-dimethoxyphenol 7c

Crude product recrystallized from chloroform-methanol to afford off white precipitate. Yield $65 \%$. Mp $148-150^{\circ} \mathrm{C}$. IR (KBr) $v_{\text {max }, 3595(O H}$ phenol $), 3077\left(\mathrm{CH}_{\mathrm{Ar}}\right), 2983,2865\left(\mathrm{CH}_{\text {aliphatic }}\right) 1618$ $(\mathrm{CH}=\mathrm{N}), 1607\left(\mathrm{C}=\mathrm{N}_{\text {oxadiazole }}\right), 1595,1986(\mathrm{C}=\mathrm{C})$, 1225(C-N), 1203(C-O), 1111 (C-O-C); cm-1 ${ }^{1}{ }^{1} \mathrm{H}-\mathrm{C} M R$ $\left.\left(400 \mathrm{MHz}, \mathrm{DMSO}-\mathrm{d}_{6}\right)\right) 1.41\left(\mathrm{t}, 3 \mathrm{H}, \mathrm{J} 6.9, \mathrm{O}-\mathrm{CH}_{2} \mathrm{CH}_{3}\right)$, $3.71\left(\mathrm{~s}, 6 \mathrm{H}, 2 \times \mathrm{OCH}_{3}\right), 3.92\left(\mathrm{q}, 2 \mathrm{H}, \mathrm{J} 7.0, \mathrm{O}-\mathrm{CH}_{2}\right)$, $4.42\left(\mathrm{~s}, 2 \mathrm{H}, \mathrm{OCH}_{2}\right), 4.45\left(\mathrm{~s}, 2 \mathrm{H}, \mathrm{OCH}_{2}\right), 6.54(\mathrm{~s}, 2 \mathrm{H}$, $\mathrm{H}-3), 6.86(\mathrm{~d}, 2 \mathrm{H}, \mathrm{J}$ 8.2, H-18), 7.24 (dd, $1 \mathrm{H}, \mathrm{J}$ 8.2; 1.7, $\mathrm{H}-19), 7.48(\mathrm{~s}, 1 \mathrm{H}, \mathrm{H}-15), 7.71 \quad(2 \mathrm{H}, \mathrm{d}, \mathrm{J} 8.24$, $\mathrm{H}-8), 8.21(2 \mathrm{H}, \mathrm{d}, \mathrm{J} 8.2, \mathrm{H}-9), 8.57(\mathrm{~s}, 1 \mathrm{H}, \mathrm{CH}=\mathrm{N})$, 9.16(bs, $1 \mathrm{H}, \mathrm{OH})$, ), $9.78(\mathrm{bs}, 1 \mathrm{H}, \mathrm{OH}),{ }^{13} \mathrm{C}-\mathrm{NMR}(100$ $\left.\mathrm{MHz}, \mathrm{DMSO}-\mathrm{d}_{6}\right): 15.2\left(1 \mathrm{C}, \mathrm{O}-\mathrm{CH}_{2} \mathrm{CH}_{3)}, 56.31(2 \mathrm{C}\right.$, $\left.\mathrm{OCH}_{3}\right), 64.2\left(1 \mathrm{C}, \mathrm{OCH}_{2}\right), 71.21\left(1 \mathrm{C}, \underline{\mathrm{CH}}_{2} \mathrm{OCH}_{2}\right)$, 69.91(1C, $\left.\mathrm{CH}_{2} \mathrm{OCH}_{2}\right), 107.85(2 \mathrm{C}, \mathrm{C}-3), 110.19(1 \mathrm{C}$, C-19), 116.25 (1C, C-15), 123.8 (1C, C-18), 126.44(1C, C-10), 129.01(2C, C-9), 129.15(1C, C14), 131.33(2C, C-8), 133.04(1C,C-1), 138.75(1C,C4), 142.07 (1C,C-7), 147.26 1C,C-16), 150.6 (1C,C- 
17) 151.89 (2C,C-2), 161.01(1C, $C=N), 163.12$ \&164.03( $\mathrm{C}=\mathrm{N}$ oxadiazole $)$. HREIMs $\mathrm{m} / \mathrm{z}=505.1845$ $\left[\mathrm{M}^{\prime+}\right]$ (calc. for $\mathrm{C}_{27} \mathrm{H}_{27} \mathrm{~N}_{3} \mathrm{O}_{7}, 505.1849$ ).

Synthesis of 4-(((4-(5-)(4-hydroxy-3,5dimethoxybenzylidene)amino)-1,3,4-oxadiazol-2yl)benzyl)oxy)methyl)-2,6-dimethoxyphenol 7d

Crude product recrystallized from ethylacetate-ethanol to afford pale yellow precipitate. Yield $62 \%$. Mp 166-168 ${ }^{\circ} \mathrm{C}$. IR (KBr) $\mathrm{v}_{\max }, 3610\left(\mathrm{OH}_{\text {phenol }}\right), 3082\left(\mathrm{CH}_{\mathrm{Ar}}\right), 2972,2860(\mathrm{CH}$ aliphatic $) 1614(\mathrm{CH}=\mathrm{N}), 1605\left(\mathrm{C}=\mathrm{N}_{\text {oxadiazole }}\right), 1595$, 1980(C=C), $1221(\mathrm{C}-\mathrm{N}), 1198(\mathrm{C}-\mathrm{O}), 1135$ (C-O-C); $\mathrm{cm}^{-1},{ }^{1} \mathrm{H}-\mathrm{NMR}\left(400 \mathrm{MHz}, \mathrm{DMSO}-\mathrm{d}_{6}\right) \delta: 3.71(\mathrm{~s}, 6 \mathrm{H}$, $\left.2 \times \mathrm{OCH}_{3}\right), 3.85\left(\mathrm{~s}, 6 \mathrm{H}, 2 \times \mathrm{OCH}_{3}\right) 4.42\left(\mathrm{~s}, 2 \mathrm{H}, \mathrm{OCH}_{2}\right)$, 4.45 (s, 2H, OCH $)_{2}, 6.57$ (s, 2H, H-3), 7.19 (s, 2H, $\mathrm{H}-15, \mathrm{H}-19), 7.75(2 \mathrm{H}, \mathrm{d}, \mathrm{J} 8.23, \mathrm{H}-8), 8.18(2 \mathrm{H}, \mathrm{d}$, J 8.2, $\mathrm{H}-9), 8.68$ (s, $1 \mathrm{H}, \mathrm{CH}=\mathrm{N}), 9.14(2 \mathrm{H}, \mathrm{bs}, 2 \times \mathrm{OH})$, ${ }^{13} \mathrm{C}-N M R\left(100 \mathrm{MHz}, \mathrm{DMSO}-\mathrm{d}_{6}\right): 56.24\left(2 \mathrm{C}, \mathrm{OCH}_{3}\right)$, 56.36 $\left(2 \mathrm{C}, \mathrm{OCH}_{3}\right), 70.77\left(1 \mathrm{C}, \mathrm{CH}_{2} \mathrm{OCH}_{2}\right), 68.92(1 \mathrm{C}$, $\left.\mathrm{CH}_{2} \mathrm{OCH}_{2}\right), 105.86$ (2C, C-3), 107.98(2C, C-15,C19), 124.8(1C, C-10), 129.21(2C, C-9), 129.15(1C, C-14), 131.33(2C, C-8), 135.04(1C, C-4), 138.75(1C, C-7), 142.4 (2C, C-1, C-17) 151.89 (4C, C-2, C-16, C-18), 160.34(1C, C=N), 162.13\&163,75 $\left(\mathrm{C}=\mathrm{N}_{\text {oxadiazole }}\right)$. HREIMs $\mathrm{m} / \mathrm{z}=521.1793\left[\mathrm{MÙ}^{+}\right]$(calc. for $\left.\mathrm{C}_{27} \mathrm{H}_{27} \mathrm{~N}_{3} \mathrm{O}_{8}, 521.1798\right)$.

Synthesis of 4-((4-(5-((4-hydroxy-3,5-di-tertbutylbenzylidene)amino)-1,3,4-oxadiazol-2yl)benzyl)oxy)methyl)-2,6-dimethoxyphenol 7e

The crude products recrystallized from Chloroform afforded pale yellow precipitate. Yield 59.7\%, mp 204-206, IR ( $\mathrm{KBr}) \mathrm{v}_{\max }, 3633.7\left(\mathrm{OH}_{\text {phenol }}\right)$ $3058\left(\mathrm{CH}_{\mathrm{Ar}}\right), 2960-2885\left(\mathrm{CH}_{\text {alph }}\right), 1621(\mathrm{CH}=\mathrm{N})$, $1601\left(\mathrm{C}=\mathrm{N}_{\text {oxadiazole }}\right), \quad 1597-1589 \quad(\mathrm{C}=\mathrm{C})$ and 1262(ArO- $\mathrm{CH}_{2}$ ) 1152(C-O-C). ' $\mathrm{H}$ NMR, $(400 \mathrm{MHz}$, $\left.\mathrm{CDCl}_{3},\right) \delta: 1.42(\mathrm{~s}, 18 \mathrm{H}, 2 \times(t-\mathrm{Bu}), 3.71(\mathrm{~s}, 6 \mathrm{H}, 2 \times$ $\left.\mathrm{OCH}_{3}\right), 4.42\left(\mathrm{~s}, 2 \mathrm{H}, \mathrm{OCH}_{2}\right), 4.45\left(\mathrm{~s}, 2 \mathrm{H}, \mathrm{OCH}_{2}\right), 5.62$ (bs, 1H, OH), 6.57 (s, 2H, H-3), 7.72 ( 2H, d, J 8.2, $\mathrm{H}-8$ ), 7.91(s, 2H, H-15,H-19), 8.18(2H, d, J 8.2, H9), 8.59(s, $1 \mathrm{H}, \mathrm{CH}=\mathrm{N}), 9.14(\mathrm{bs}, 1 \mathrm{H}, \mathrm{OH}),{ }^{13} \mathrm{C}-\mathrm{NMR}$ (100 MHz, DMSO- $\left.\mathrm{d}_{6}\right): 30.32\left(6 \mathrm{C}, 4\left(\mathrm{C}\left(\mathrm{CH}_{3}\right)_{3}\right)\right.$, $\left.\left.34.56\left(2 \mathrm{C}, \underline{\mathrm{C}}\left(\mathrm{CH}_{3}\right)_{3}\right),\right)\right) 56.12\left(2 \mathrm{C}, \mathrm{OCH}_{3}\right), 71.21(1 \mathrm{C}$, $\left.\underline{\mathrm{CH}}_{2} \mathrm{OCH}_{2}\right), 69.91\left(1 \mathrm{C}, \mathrm{CH}_{2} \mathrm{OCH}_{2}\right), 107.85(2 \mathrm{C}, \mathrm{C}-3)$, 123.76 (2C, C-15, C-19) , 124.81 (1C, C10), 129.01(2C, C-9), 129.15(1C,C-14), 131.33(2C, C$8), 134.04(1 \mathrm{C}, \mathrm{C}-4), 136.17(2 \mathrm{C}, \mathrm{C}-16, \mathrm{C}-$ 18) $138.44(1 \mathrm{C}, \mathrm{C}-1), 142.07$ (1C, C-7) 151.89 (2C,
C-2), 157.25 (1C, C-17), $161.44(1 \mathrm{C}, \mathrm{C}=\mathrm{N})$, $162.44 \& 165.11(\mathrm{C}=\mathrm{N}$ oxadiazole $)$. HREIMs $\mathrm{m} / \mathrm{z}=$ 573.2833 [MÙ'] (calc. for $\mathrm{C}_{33} \mathrm{H}_{39} \mathrm{~N}_{3} \mathrm{O}_{6}, 573.2839$ ).

\section{Antioxidant \\ DPPH assay}

The assay was performed as reported by Gerhauser et al. ${ }^{27}$. Five microliters of the sample (dissolved in ethanol) was added into $195 \mu \mathrm{L}$ of $100 \mu \mathrm{M}$ DPPH reagent in ethanol (96\%) and mixed in a 96-well plate. The intensity of the color was measured for $3 \mathrm{~h}$ at an interval of $20 \mathrm{~min}$ at $515 \mathrm{~nm}$. Ascorbic acid and BHT were used as reference

\section{FRAP assay}

The FRAP assay was performed according to the Benzie and Strain ${ }^{28}$ method. The FRAP reagent was prepared by combining $300 \mathrm{mM}$ acetate buffer and $10 \mathrm{mM}$ 2,4,6-tripyridyl-s-triazine (TPTZ) solution in $40 \mathrm{mM} \mathrm{HCl}$ and $20 \mathrm{mM}$ $\mathrm{FeCl} 3 \cdot 6 \mathrm{H} 2 \mathrm{O}$, in a ratio of 10:1:1. The FRAP reagent was incubated at $37^{\circ} \mathrm{C}$ prior to use. Ten microliters of the sample was reconstituted in the carrier (solvent or ultrapure water) and mixed with $300 \mu \mathrm{L}$ of FRAP reagent. The mixture was incubated at 37 ${ }^{\circ} \mathrm{C}$ for $4 \mathrm{~min}$ in a microplate reader. The absorbance of the complex was $593 \mathrm{~nm}$. The FRAP value can be calculated using the following equation ${ }^{29}$ :

FRAP $=[(0-4 \mathrm{~min}$ "A593 $\mathrm{nm}$ of test sample $) /(0-4$ min "A593 $\mathrm{nm}$ of standard)]

$$
\times[\text { standard }](\mu \mathrm{M}) \times \mathrm{Y} \times 1000
$$

Where

$\mathrm{Y}$ is the absorbance of the spectrophotometer.

\section{RESULTS AND DISCUSSION}

\section{Chemistry}

The new compounds (7a-7e) were synthesized from multi-stapes. Scheme 1 displayed the synthesise of 5-dimethyl-4-((trimethylsilyl)oxy) benzyl alcohol (2) which is used as starting material to synthesis of 4-(( (3,5-dimethoxy-4((trimethylsilyl)oxy)benzyl)oxy)methyl) benzonitrile (3) as depicted in Scheme 2.

Compound $\mathbf{3}$ was converted to the corresponding acid 4 then the acid to the 
corresponding hydrazide (5). The hydrazide cyclized to the 5-amino-1,3,4-oxadiazole 6 . The 5amino-1,3,4-oxadiazole were reacted with several substituted of 4-hydroxybenzylaldehyde (Table 1 ) as shown in Scheme 2.

All synthesized compounds were characterized from their IR, ${ }^{1} \mathrm{H}-\mathrm{NMR},{ }^{13} \mathrm{C}-\mathrm{NMR}$ spectrum beside the EIMS and HRMIS.

The IR spectrum of compound $\mathbf{3}$, showed an interesting peak at $2248 \mathrm{~cm}^{-1}$ for Ca"N which is disappearing from compound 4 and a new peak of $\mathrm{C}=\mathrm{O}$ was appeared at $1673 \mathrm{~cm}^{-1}$, as well interesting peak of $\mathrm{C}=\mathrm{N}$ of the oxadiazole ring for compound 6 was appearing at $1609 \mathrm{~cm}^{-1}$. Moreover, the $\mathrm{C}=\mathrm{N}$ of Schiff bases of compounds 7a-7e were appeared at $1614-1621 \mathrm{~cm}^{-1}$ beside the $\mathrm{C}=\mathrm{N}$ of the oxadiazole ring. The ${ }^{1} \mathrm{H}-\mathrm{NMR}$ spectrum of compound 2 showed the disappearing of the proton of aldehyde from $9.85 \mathrm{ppm}$ and appearing of two protons of $\mathrm{CH}_{2}$ beside the $\mathrm{OH}$ which represents the successful reduction of aldehyde group. Furthermore, the new two doublets for compound 3 at 7.41 and 7.49 ppm
( $\mathrm{H}-8, \mathrm{H}-9$ ) with copling constant (J) 8.2 and $7.9 \mathrm{~Hz}$ respectively represented successfully of reaction compound 2 with 4-(bromo methyl)benzonitrile. As well ${ }^{1} \mathrm{H}$-NMR of compound 4 showed disappearing of trimethyl Isilyl grop from 0.18 ppm and appears of the broad singlet of $\mathrm{OH}$ for the converting the nitrile group to carboxylic acid. The ${ }^{1} \mathrm{H}-\mathrm{NMR}$ spectrum of compound $\mathbf{5}$ showed the disappearing of $\mathrm{H}$ of carboxylic and appeared of new two board singlet of $\mathrm{NH}_{2}$ and $\mathrm{NH}$ at 4.74 and 8.83 ppm respectively, while compound 6 showed disappear these peaks and appeared new signal at 7.51 which is represented the $\mathrm{NH}_{2}$ attached the oxadiazole ring. The ${ }^{1} \mathrm{H}-\mathrm{NMR}$ of compounds $7 \mathrm{a}-7 \mathrm{e}$ showed appearance of new peaks represented the Schiff base (Imin proton) at 8.57-8.68 ppm. The peaks of the substituted 4-hydroxylphenol appeared in their expected areas (see the experimental section). The ${ }^{13} \mathrm{C}-\mathrm{NMR}$ spectrum of compound 2 showed the carbons of trimethylsilyl at -0.05 as well the carbon of $\mathrm{CH}_{2}-\mathrm{OH}$. While the ${ }^{13} \mathrm{C}-\mathrm{NMR}$ spectrum of compound 3 displayed two carbons of $\mathrm{CH}_{2} \mathrm{OCH}_{2}$ at 70.76 \& 71.14 beside the Ca"N at 101.22 also the carbons of benzonitrile ring. Disappearing of carbon

Table 1: The substituent group and selected properties of synthesized compound 7a-7e

\begin{tabular}{llllccc}
\hline No. & $\mathbf{R}_{\mathbf{1}}$ & $\mathbf{R}_{\mathbf{2}}$ & Yield \% & M.P.C & HREIMS calc. & HREIMS Found \\
\hline $7 \mathrm{a}$ & $\mathrm{H}$ & $\mathrm{H}$ & 72 & $179-181$ & 461.1587 & 461.1581 \\
$7 \mathrm{~b}$ & $\mathrm{H}$ & $\mathrm{OCH}_{3}$ & 69 & $122-124$ & 491.1693 & 491.1690 \\
$7 \mathrm{c}$ & $\mathrm{H}$ & $\mathrm{OEt}$ & 65 & $148-150$ & 505.1849 & 505.1845 \\
$7 \mathrm{~d}$ & $\mathrm{OCH}_{3}$ & $\mathrm{OCH}_{3}$ & 62 & $166-168$ & 521.1798 & 521.1793 \\
$7 \mathrm{e}$ & $\mathrm{C}\left(\mathrm{CH}_{3}\right)_{3}$ & $\mathrm{C}\left(\mathrm{CH}_{3}\right)_{3}$ & 60 & $204-206$ & 573.2839 & 573.283 \\
\hline
\end{tabular}

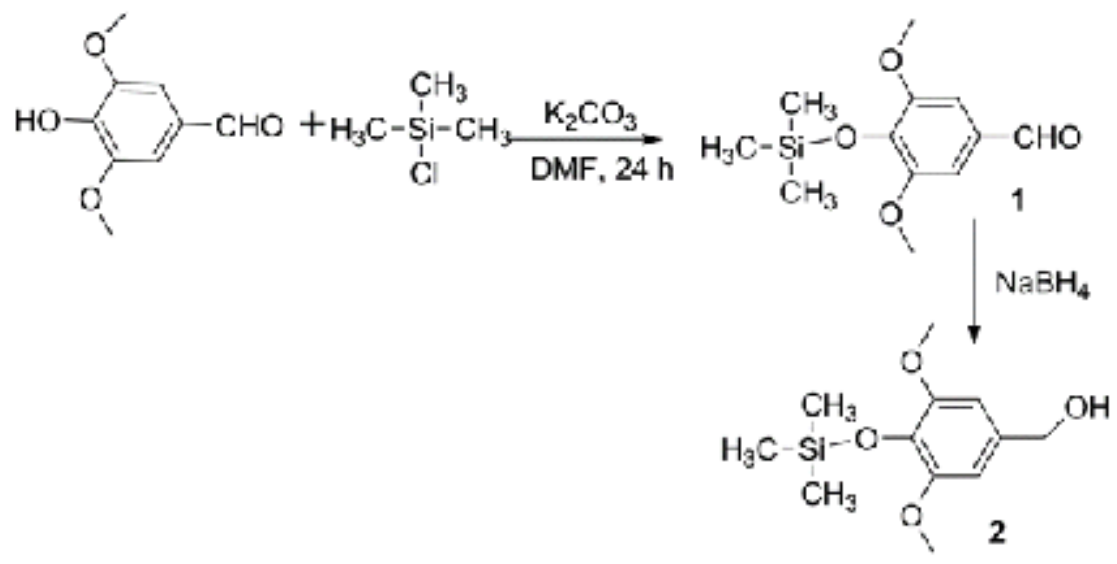

Scheme 1: Synthetic route of 3,5-dimethyl-4-((trimethylsilyl)oxy)benzyl alcohol 

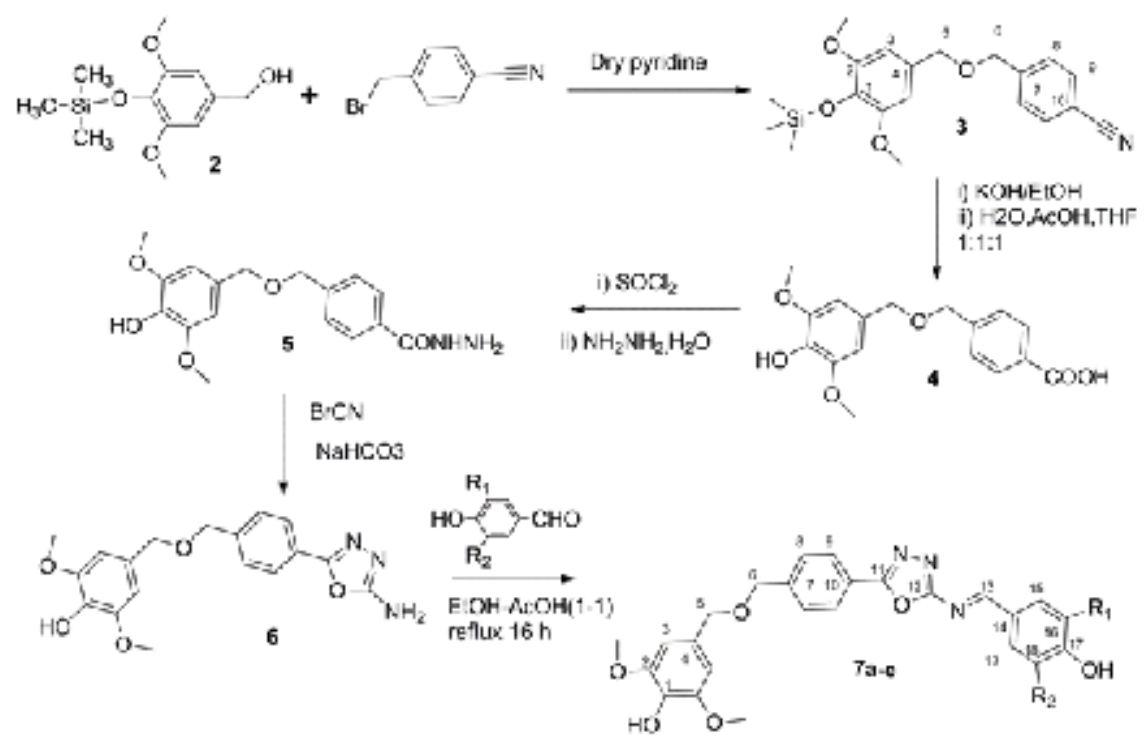

Scheme 2: Synthetic route of synthesis 4-(((4-(5-)((aryidene) amino)-1,3,4-oxadiazol-2-yl)benzyl)oxy) methyl)-2,6-dimethoxyphenol

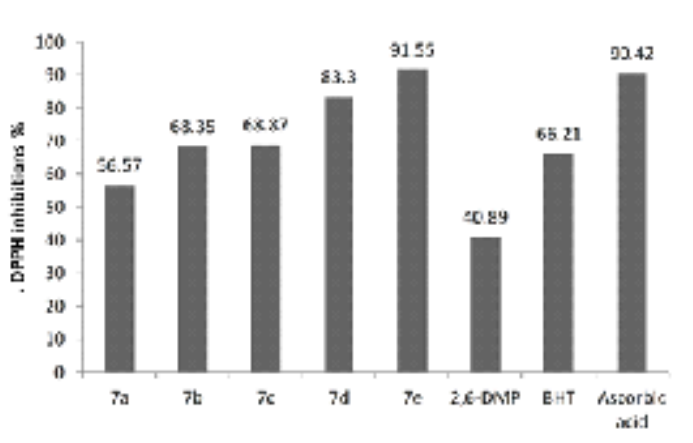

Fig. 1: DPPH inhibitions $\%$ of $7 a-7 e$

Ca" $\mathrm{N}$ and raised a new carbon of $\mathrm{C}=\mathrm{O}$ that Enhances the evidence of successful convert compound $\mathbf{3}$ to $\mathbf{4}$ besides the IR and ${ }^{1} \mathrm{H}-\mathrm{NMR}$. The ${ }^{13} \mathrm{C}-\mathrm{NMR}$ of compound 6 showed two carbons of $\mathrm{C}=\mathrm{N}$ of oxadiazole ring instead of the carbon of the carbonyl group. The ${ }^{13} \mathrm{C}$ spectra of compounds 7a7e were showed an interested peak of $\mathrm{CH}=\mathrm{N}$ beside the carbons of the substitute 4hydroxyphenol. As well as all the substituent group for $7 \mathbf{b}-7 \mathbf{e}$ were appeared in the expected range. The EIMS spectra showed the molecular ion $\mathrm{M}^{*+}$ for all compounds and the base peak (100\%) as well<smiles></smiles>

Fig. 2: The possible resonance structure 


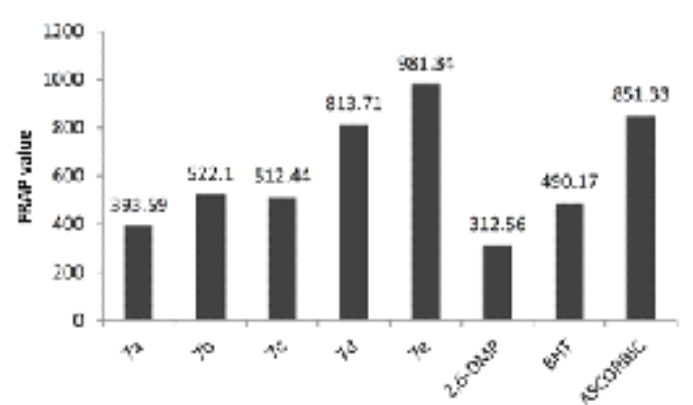

Fig. 3: FRAP value of $7 a-7 e$

the HREIMs value was confirmed the accurate mass and the molecular formula as depicted in Table 1.

\section{Antioxidant activity}

The synthesized compounds (7a-7e) were exhibited high antioxidant ability in both assaysBased on the type of substitute group of phenol. Compound 7e, which I possess 2,6 di-tertbutylphenol group shows antioxidant ability slightly higher than ascorbic acid in both assays (DPPH and FRAP) as shown in Figure 1

While, compound $\mathbf{7 d}$ which is posses 2,6di methoxy phenol group exhibited antioxidant ability higher than BHT also, all compounds showed antioxidant activity more than 2,6-dimethoxy phenol itself. The antioxidant of the substituted phenol followed the following sequence: 2,6-di-tertbutyl>2,6-dimethoxy>2-methoxyH"2-ethoxy> non substituted phenol. These results are in agreement with many articles $x x x$ that the more hindered phenol increase the antioxidant stability. Furthermore, we assume that the long chain resonance between the substituted phenol and the main group $(\mathrm{CH}=\mathrm{N})$ attached the oxadiazole ring after donating a proton could enhance the free radical stability, which can enhance the free-radical scavenging ability. As shown in Figure 2.

The FRAP value was higher than DPPH when comparing the results with the references and this difference could be attributed to the different mechanisms for FRAP and DPPH. FRAP involves a single electron transfer mechanism, whereas DPPH assay depends on the $\mathrm{H}$-atom transfer mechanism ${ }^{30}$ beside the hindrances around the phenol. Figure 3 displayed the FRAP value of the synthesized compound 7a-7e

\section{CONCLUSIONS}

A series of new Schiff base compounds from 5amino-1,3,4-oxadizole incorporating hindered phenol moieties were successfully synthesized and characterized. All of the new compounds were screened for antioxidant activity using the FRAP and DPPH assays. The antioxidant ability of these compounds increases with increasing the hindered phenol.

\section{ACKNOWLEDGEMENTS}

The author would like to thank the University of Malaya for running the NMR We are also grateful to Mr. Mohammed Farouq Halabi, Department of Biomedical Science, Faculty of Medicine, University of Malaya for his great help with screening the antioxidant and Dr.Raied M. Shakir for his contributions as well, we would like to Acknowledge NUS, Singapore for running the EIMS and HREIMS. Also, we would like to thank the University of Baghdad for supporting this study and provide the grant for this study.

\section{REFERENCES}

1. Shacter, E. Drug Metabolism Reviews 2000, 32, 307.

2. Chatterjee, R.; Bandyopadhyay, U.; Mazumdar, A.; Banerjee, R. K. Biochem Pharmacol. 1996, 52, 1169.

3. Sangeetha, P.; Das, U. N.; Koratkar, R.; Suryaprabha, P. Free Radic Biol Med. 1990, 8, 15.
4. Kar, S., Subbaram, S., Carrico, P M., Melendez, J A. Respir Physiol Neurobiol. 2010, 174, 299.

5. Khalil, Z.; Khodr, B. Free Radic Biol Med. 2001, 31, 430.

6. Mullican, M. D.; Wilson, M. W.; Connor, D. T.; Kostlan, C. R.; Schrier, D. J.; Dyer, R. D. J. Med. Chem 1993, 36, 1090. 
7. Ye, X.; Zhou, W.; Li, Y.; Sun, Y.; Zhang, Y.; Ji, H.; Lai, Y. Cancer Chemotherapy and Pharmacology 2010, 66, 277.

8. YEN, C.-Y. H. A. G.-C. J. Agric. Food Chem. 2002, 50, 2993.

9. Shakir, R. M.; Ariffin, A.; Abdulla, M. molecules 2014, 19, 3436.

10. Jeong, J.-M.; Kang, S.-K.; Lee, I.-H.; Lee, J.Y.; Jung, H.; Choi, C.-H. J Pharm Pharmaceut Sci 2007, 10, 537.

11. Edge, R.; McGarvey, D. J.; Truscott, T. G. J Photoch Photobio B 1997, 41, 189.

12. Sreeramulu, J.; Ashokgajapathiraju, P. Orient J Chem 2014, 30, 651.

13. SHAH, H. P., SHAH, B. R., BHATT, J. J., DESAI, N. C., TRIVEDI, P. B. and UNDAVIA, N. K. Indian J. Chem 1998, 37, 180.

14. de Oliveira, C. S.; Lira, B. F.; Barbosa-Filho, J. M.; Lorenzo, J. G. F.; de Athayde-Filho, P. F. molecules 2012, 17, 10192.

15. Musad, E. A.; Mohamed, R.; Ali Saeed, B.; Vishwanath, B. S.; Lokanatha Rai, K. M. Bioorganic \& Medicinal Chemistry Letters 2011, 21, 3536.

16. Radhika Maheshwari, P. C., Shubhini Saraf Medicinal Chemistry Research 2010, 1.

17. Ogiri, S.; Ikeda, M.; Kanazawa, A.; Shiono, T.; Ikeda, T. Polymer 1999, 40, 2145.

18. Liu, H.; Fu, Z.-e.; Xu, K.; Cai, H.-I.; Liu, X.; Chen, M.-C. Materials Chemistry and Physics 2012, 132, 950.

19. Shan, X.; Ibrahim, A. O.; Zhou, Y.; Zhang, H.; Ma, J.; Jiang, F.; Hong, M. Inorganic Chemistry Communications 2012, 22, 149.

20. Kim, S.-H.; Gwon, S.-Y.; Burkinshaw, S. M.;
Son, Y.-A. Dyes and Pigments 2010, 87, 268.

21. Chandra, M.; Sahay, A. N.; Pandey, D. S.; Tripathi, R. P.; Saxena, J. K.; Reddy, V. J. M.; Carmen Puerta, M.; Valerga, P. Journal of Organometallic Chemistry 2004, 689, 2256.

22. Bodtke, A.; Pfeiffer, W.-D.; Ahrens, N.; Langer, P. Bioorganic \& Medicinal Chemistry Letters 2004, 14, 1509.

23. Kaya, 0.; Cihangirolu, N. Journal of Polymer Research 2004, 11, 37.

24. Khodair, A. I.; Bertrand, P. Tetrahedron 1998, 54, 4859.

25. Huihui Ti; Qing Li; Ruifen Zhang; Mingwei Zhang; Yuanyuan Deng; Zhencheng Wei; Jianwei Chi; Yan Zhang Food Chemistry 2014, 159166.

26. Fausta Natella, M. N., Maurizio Di Felice, and Cristina Scaccini*; Free Radical Research Group, N. I. o. N., Roma, Italy J. Agric. Food Chem. 1999, 47, 1453.

27. Gerhäuser, C.; Klimo, K.; Heiss, E.; Neumann, I.; Gamal-Eldeen, A.; Knauft, J.; Liu, G.-Y.; Sitthimonchai, S.; Frank, N. Mutation Research/Fundamental and Molecular Mechanisms of Mutagenesis 2003, 523-524, 163.

28. Benzie, I. F.; Strain, J. J. Anal. Biochem. 1996, 239, 70.

29. Rustaiyan, A., Javidnia, K., Farjam, M H., Aboee-Mehrizi, F., E. J Med Plants Res. 2011, 5, 4251.

30. Huang, D.; Ou, B.; Prior, R. L. Journal of Agricultural and Food Chemistry 2005, 53, 1841. 